\title{
Protocol for a randomized controlled trial to test the acceptability and adherence to 6-months of walnut supplementation in Chinese adults at high risk of cardiovascular disease
}

Yishu Liu ${ }^{1,2}$, Nan Li ${ }^{3}$, Ni Yan ${ }^{3}$, Xiong-fei Pan ${ }^{1}$, Qiang Li ${ }^{1}$, Renata Micha ${ }^{4}$, Dariush Mozaffarian ${ }^{4}$, Mark D. Huffman ${ }^{1,5}$, Yanfang Wang ${ }^{6}$, Bruce Neal ${ }^{1,7,8}$, Maoyi Tian ${ }^{1,2}$, Yi Zhao ${ }^{3^{*}}$ and Jason H. Y. Wu ${ }^{1 *}$ (D)

\begin{abstract}
Background: Consumption of nuts improves cardio-metabolic risk factors in clinical trials and relates to lower risk of cardiovascular disease (CVD) in prospective observational studies. However, there has not been an adequately powered randomized controlled trial to test if nuts supplementation actually reduces incident CVD. In order to establish the feasibility of such a trial, the current study aimed to assess the acceptability and adherence to longterm nut supplementation amongst individuals at high CVD risk in China.
\end{abstract}

Methods: This protocol described a 6-month trial performed in Ningxia Province in China among participants with a history of CVD or older age (female $\geq 65$ years, male $\geq 60$ years) with multiple CVD risk factors. Participants were randomized to control (received non-edible gift), low dose walnut $(30 \mathrm{~g} / \mathrm{d})$, or high dose walnut $(60 \mathrm{~g} / \mathrm{d}) \mathrm{groups}$ in a 1:1:1 ratio. Walnuts were provided at no cost to participants and could be consumed according to personal preferences. Follow-up visits were scheduled at 2 weeks, 3 months and 6 months. The primary outcome was fasting plasma alpha linolenic acid (ALA) levels used as an indicator of walnut consumption. Secondary outcomes included self-reported walnut intake from the $24 \mathrm{~h}$ dietary recalls. The target sample size of 210 provided $90 \%$ statistical power with two-sided alpha of 0.05 to detect a mean difference of $0.12 \%$ (as percent of total fatty acid) in plasma ALA between randomized groups.

Results: Two hundred and ten participants were recruited and randomized during October 2019. Mean age of participants was 65 years ( $S D=7.3$ ), 47\% were females, and 94\% had a history of CVD at baseline. Across the three study groups, participants had similar baseline demographic and clinical characteristics.

(Continued on next page)

\footnotetext{
*Correspondence: zhaoyi751114@gmail.com; jwu1@georgeinstitute.org.au

${ }^{3}$ Ningxia Medical University, Yinchuan, China

${ }^{1}$ The George Institute for Global Health, Faculty of Medicine, University of New South Wales, Sydney, Australia

Full list of author information is available at the end of the article
}

C C The Author(s). 2021 Open Access This article is licensed under a Creative Commons Attribution 4.0 International License, which permits use, sharing, adaptation, distribution and reproduction in any medium or format, as long as you give appropriate credit to the original author(s) and the source, provide a link to the Creative Commons licence, and indicate if changes were made. The images or other third party material in this article are included in the article's Creative Commons licence, unless indicated otherwise in a credit line to the material. If material is not included in the article's Creative Commons licence and your intended use is not permitted by statutory regulation or exceeds the permitted use, you will need to obtain permission directly from the copyright holder. To view a copy of this licence, visit http://creativecommons.org/licenses/by/4.0/ The Creative Commons Public Domain Dedication waiver (http://creativecommons.org/publicdomain/zero/1.0/) applies to the data made available in this article, unless otherwise stated in a credit line to the data. 
(Continued from previous page)

Discussion: This trial will quantify acceptability and adherence to long-term walnut supplementation in a Chinese population at high risk of CVD. The findings will support the design of a future large trial to test the effect of walnut supplementation for CVD prevention.

Trial registration: NCT04037943

Protocol version: v3.0 August 142019

Keywords: Nuts, Cardiovascular disease, Randomized controlled trial, Plasma ALA,

\section{Background}

Tree nuts (referred to hereafter as nuts) such as walnuts, almonds, and pistachios are rich in unsaturated fats, soluble fibre, phytosterols, and antioxidants. In metaanalyses of short-term randomized controlled trials (RCT), increased intake of nuts improves cardiovascular disease (CVD) risk factors such as total cholesterol, LDL cholesterol, apolipoprotein B, and triglycerides, as well as markers of endothelial function and glycemic control [1-3]. Unsaturated fats - about $50 \%$ of total fats in most nuts - also significantly improve glycemic control in RCTs [4, 5]. These effects appear dependent on the amount (dose) rather than the types of nuts, although most studies to date have focused on walnuts and almonds [1]. Prospective observational studies also support a beneficial role of nuts for the prevention of CVD. A recent meta-analysis has found that higher nuts intake is associated with lower risk of incident CVD, with about $20 \%$ lower risk per daily $1-\mathrm{oz}(28 \mathrm{~g})$ serving (RR: 0.79, 95\% CI: 0.70, 0.88) [6].

While the findings from short-term trials and prospective cohorts suggest likely metabolic benefits and protection against CVD, and a growing number of dietary guidelines around the world recommend the incorporation of nuts as part of a healthy dietary pattern [7-9], several key questions remain unresolved. First, no longterm RCTs have tested the effects of nuts consumption on the risk of CVD. The PREDIMED study, a RCT among Spanish adults, has found that advice to consume a Mediterranean diet supplemented with mixed nuts (30 g/day) significantly reduced incident CVD, compared to advice to follow a low-fat diet [10]. However, the design of PREDIMED does not allow separation of the effects of nuts alone, vs. the Mediterranean dietary advice as a whole. In addition, errors and inconsistencies in the randomization process of PREDIMED, have raised controversy over its findings [11]. Crucially, nearly all the prior trials and cohort studies, including the PREDIMED trial, have been performed in high-income Western populations. The effects of increased nuts intake on CVD in lower- and middle-income countries remain uncertain. For instance, only three controlled trials have been conducted in Chinese participants ( 2 in China, 1 in Taiwan) [12-14], with several additional trials in other Asian populations $[15,16]$. Their findings support likely benefits of nuts supplementation in reducing LDL cholesterol and improving other CVD risk factors. However, all of the trials were short-term (less than 3 months) and not able to test for clinical CVD events.

Cardiovascular disease is the leading cause of death and disability in China [17]. Large dietary surveys indicate that low levels of nuts consumption could be a significant contributor to CVD burden in China, with estimated mean nuts intake of only $5.4 \mathrm{~g} /$ day across diverse geographical regions [18]. An appropriately designed and powered RCT to test the effect of nuts supplementation on the risk of incident CVD events in China is therefore of significant scientific and public health importance. We hereby report the protocol for a 6-month walnut supplementation trial in a rural area of China with high rates of CVD, to obtain data on feasibility of long-term walnut supplementation to support and help to refine the design of a future long-term large RCT testing the effect of nuts supplementation on CVD risk. The main objectives of the current trial are to assess the acceptability and adherence to two different doses of walnut supplementation (30 and $60 \mathrm{~g}$ per day). The exploratory objectives are to determine the effects of walnut supplementation on cardiovascular risk factors including blood lipids, fasting glucose, and body weight.

\section{Methods \\ Study design}

The 'Nuts for the prevention of cardiovascular disease in Chinese adults' (NUTS) study was a randomized, controlled, single blind (blinded investigators and blinded assessments of all study endpoints), three-group parallel design trial lasting for 6 months.

Participants were randomized to one of the three groups in a 1:1:1 ratio: control (non-edible gift), low dose (30 g/day) walnut intervention group, and high dose (60 g/day) walnut intervention group (Fig. 1). Written informed consent was obtained from each study participant.

\section{Study population}

We have chosen Ningxia to conduct this trial, since it is a province with high prevalence and mortality rate of 


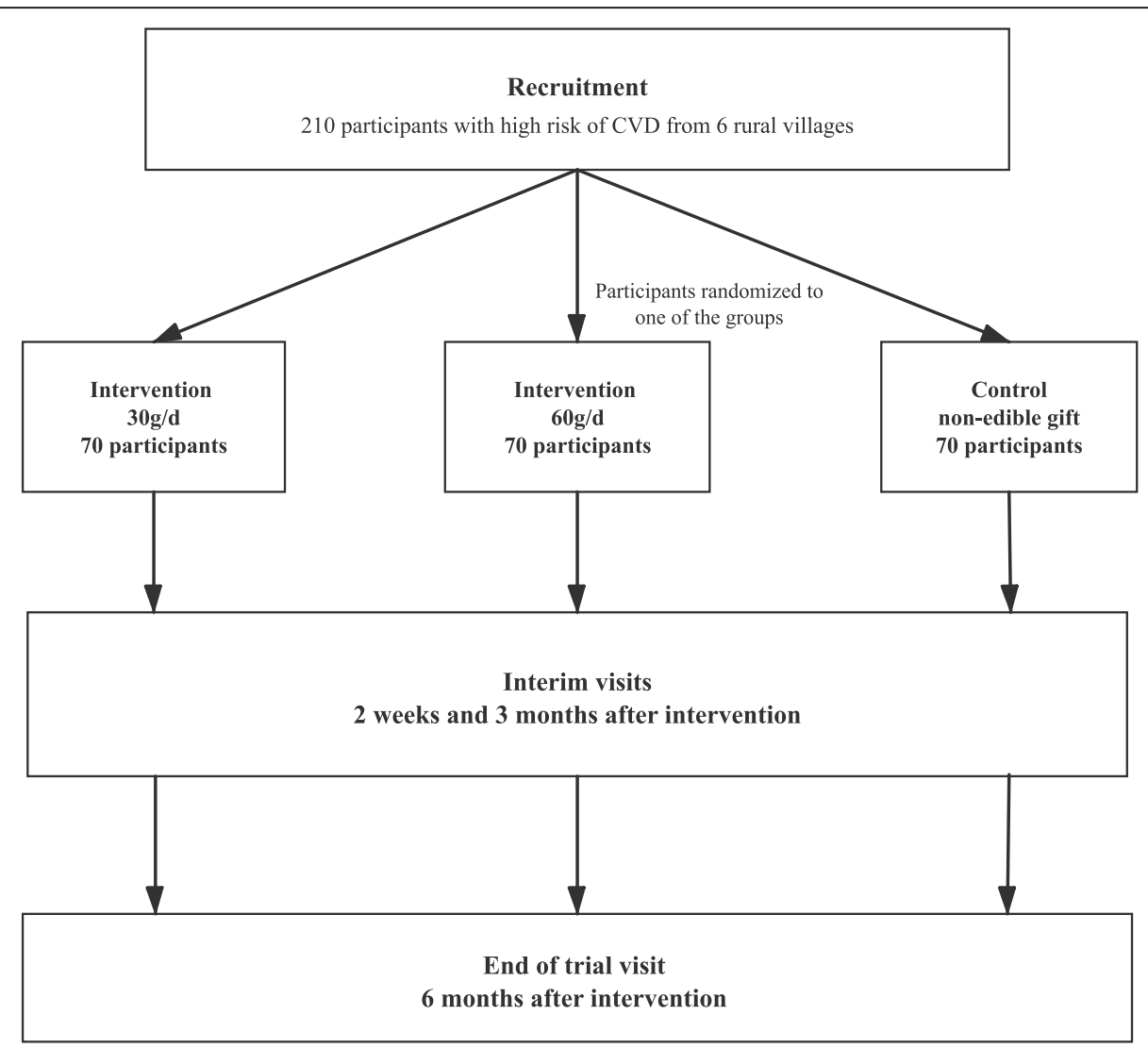

Fig. 1 Trial Flowchart. 210 participants with high risk of CVD were recruited from 6 rural villages in Ningxia Province, China. They were randomized in a 1:1:1 ratio to either the control group, or low-dose walnut (30 g/day) intervention group, or high-dose walnut (60 g/day) intervention group; and followed-up for 6 months. Outcome data were collected from participants at study baseline, the interim visits, and at the end of trial visit

stroke and ischemic heart disease [19-21] and the project team has established collaborations with local academic and government institutions. Following consultation with these partners, we recruited adults from six rural villages near Qingtongxia City in Ningxia, based on their willingness to participate and their geographic accessibility. Suitable villages needed to have at least one village doctor who was able to facilitate primary screening of participants.

In each village, we recruited individuals at high risk of CVD based upon having a history of CVD or a high risk of CVD. Inclusion criteria were a history of CVD (defined by prior hospitalization for a myocardial infarction or stroke, unstable angina, coronary artery bypass graft surgery, percutaneous coronary intervention, peripheral revascularization, symptomatic with documented hemodynamically-significant carotid or peripheral vascular disease, or amputation secondary to vascular disease); or older age ( $\geq 60$ for males, $\geq 65$ years for females), plus at least two of the following CVD risk factors: 1) type 2 diabetes including treatment with at least two oral anti-hyperglycaemic agents, or insulin, 2) systolic blood pressure $\geq 140$ $\mathrm{mmHg}$ while on one or more antihypertensive agents, 3) current daily smoking, 4) dyslipidaemia defined as HDL-cholesterol $<1.0 \mathrm{mmol} / \mathrm{L}$ or LDL-cholesterol > $6.0 \mathrm{mmol} / \mathrm{L}$, or 5) micro- or macro-albuminuria. The definition of high risk individuals was selected to ensure adequate numbers of events and statistical power in the future planned large-scale trial to test walnut supplementation to reduce CVD events. Data from completed and ongoing large-scale trials indicated that patients meeting these criteria experienced an annual event rate of $3.5 \%$ for the occurrence of major adverse cardiovascular events [22, 23].

Screening of CVD risk factors was based on self-report with support of physical evidence. Participants were deemed to have the relevant risk factors if they were able to present evidence from a laboratory measurement, prescription drug packaging, tobacco packaging, or clinical report within the past 12 months.

Individuals were excluded if they had never or rarely consumed any types of nuts, had self-reported allergy to nuts (i.e. history of food allergy with hypersensitivity to 
any of the components of nuts), had other serious medical conditions that prevent nuts consumption (e.g. digestive disease with fat intolerance), had any medical condition thought to limit survival to less than 1 year, had difficulty with consuming nuts (e.g. dental health issues that prevent chewing walnuts), or were unwilling to consume nuts.

\section{Recruitment and consent}

Village doctors distributed study information sheets in their villages and registered individuals who were interested to participate 2 weeks before study recruitment. Potential participants were invited by the village doctors to attend the screening interview at the village clinics. At the screening interview, potential participants were interviewed by trained study staff who explained the background, objectives, and procedures of the trial, sought and obtained written informed consent, confirmed eligibility, and completed the baseline survey. Participants who provided informed consent were invited to complete the baseline fasting blood sample collection the next day. Only one eligible participant was recruited from each household to avoid potential contamination across randomized groups.

\section{Randomization}

Randomization was done centrally by an independent biostatistician using a computer-generated random number sequence after the completion of the baseline survey. Participants were randomized with stratification by village in a 1:1:1 allocation ratio to either one of the intervention groups or to the control group.

\section{Rationale for the chosen walnut intervention}

Walnuts contain a high amount of protein, dietary fibre, vitamins, minerals and phytosterols [24]. Walnuts are a rich source of both omega- 6 and omega- 3 polyunsaturated fatty acids (linoleic and alpha linolenic acid [ALA]) [25] and improve cardio-metabolic risk factors in shortterm clinical feeding trials including lowering LDLcholesterol [26, 27]. Walnuts have made up the largest proportion $(50 \%)$ of the mixed nuts used in the PREDIM ED study [10]. In addition, walnuts are relatively soft compared to other nut types, making them easier to be chewed and consumed by those with oral health issues such as older adults, who are likely to make up a significant proportion of those recruited to the trial. Walnuts are also readily accessible since China grows a large volume of walnuts [28]. Commercially-available roasted, unsalted walnuts were used in the trial for enhanced taste and longer shelf life. The study walnuts contained $4.47 \mathrm{~g}$ of protein, $17.64 \mathrm{~g}$ of total fat, $5.73 \mathrm{~g}$ of carbohydrate and total energy of $811 \mathrm{KJ}$ per $30 \mathrm{~g}$.
A dose-response relationship between greater nuts intake and lower cardio-metabolic risk has been generally observed across a range of nuts intake between $5 \mathrm{~g}$ to $100 \mathrm{~g}$ per day, with stronger effects for trials providing $\geq 60 \mathrm{~g} /$ day [1, 2]. Since the effect of walnut intake on CVD risk is likely mediated by changes in cardiometabolic risk factors, we seek to evaluate if a higher dose of daily walnut supplementation is as acceptable as a lower dose. Therefore, for this trial, we tested both 30 g per day (corresponding to many current dietary recommendations), as well as a higher dose of $60 \mathrm{~g}$ per day of walnut supplementation.

\section{Intervention and control}

Participants in the intervention groups were provided sachets containing $30 \mathrm{~g}$ of roasted and unsalted walnuts free of charge. For the $30 \mathrm{~g} /$ day arm, 1 sachet/day was provided, and for the $60 \mathrm{~g} /$ day arm, 2 sachets/day were provided. The walnuts were purchased from a local food manufacturer who sourced walnuts from Chinese walnut growers and packaged them in accordance with Chinese food standards (GB/T 20398-2006). The participants were instructed to open and consume the allocated dose of walnuts at any time of the day according to their personal preference, but not to share the walnuts with family members. To ensure freshness, participants were provided with sufficient sachets for the first 3 months of the intervention at baseline and were provided with the remainder at the 3-month follow-up study visit.

Participants in the control group continued with their usual diet without introduction of any nuts supplement. They were provided with small, inedible toiletry gifts at baseline and were provided with similar gifts at each follow-up visit to ensure equal study contacts across all the study groups and encourage attendance at the follow-up visits.

\section{Data collection and follow up}

Informed consent was obtained from each participant before any data collection (Table 1). At baseline, eligible participants completed an interviewer-administered questionnaire, a validated 95-item food frequency questionnaire (FFQ) that asked participants about their usual dietary pattern over the past 12 months, and a 24-h dietary recall [29]. Each participant also underwent physical examination to record height, weight, waist and hip circumferences (Inbody-370S scale and measuring tapes, and anthropometry assessment conducted according to the International Society for the Advancement of Kinanthropometry (ISAK) protocols), and blood pressure (OMRON-7124). After an overnight fast, participants had their blood samples collected at the local township hospital by trained nurses. One blood sample was drawn into $5 \mathrm{ml}$ EDTA-anticoagulated vacutainer tube, and 
Table 1 Timeline for key events and collection of outcome data for the NUTS trial

\begin{tabular}{|c|c|c|c|c|c|c|c|}
\hline & & & Follow up & eks & & & \\
\hline & & & Baseline & & & & \\
\hline Week & -2 & -1 & 0 & 1 & 2 & 12 & $25^{\mathrm{a}}$ \\
\hline Months & & & 0 & & & 3 & 6 \\
\hline Pre-intervention screening & & & & & & & \\
\hline Information sheets distribution & X & & & & & & \\
\hline Consent for pre-screening & x & X & & & & & \\
\hline Pre-screening & X & X & & & & & \\
\hline Baseline & & & & & & & \\
\hline Informed consent & & & X & & & & \\
\hline Assessed for eligibility & & & X & & & & \\
\hline Demographics & & & X & & & & \\
\hline Disease history & & & X & & & & \\
\hline Medication use & & & X & & & & \\
\hline Food Frequency Questionnaire & & & X & & & & \\
\hline Intervention & & & & & & & \\
\hline Randomization & & & & X & & & \\
\hline Dispense walnuts (intervention groups) & & & & x & & x & \\
\hline Dispense non-edible gifts (control group) & & & & X & X & X & X \\
\hline Endpoint collection & & & & & & & \\
\hline Blood pressure & & & X & & & & X \\
\hline Fasting blood & & & X & & & & x \\
\hline Anthropometric measurements ${ }^{\mathrm{b}}$ & & & x & & & & x \\
\hline 24-h dietary recall & & & x & & & & X \\
\hline Adherence and acceptability (intervention groups) & & & & & X & X & x \\
\hline
\end{tabular}

used for measurement of blood lipids (total triglycerides, total cholesterol, LDL-C, and HDL-C) at the township hospital laboratory immediately following routine procedures for the results to be provided to the participants. Another blood samples were drawn into $5 \mathrm{ml}$ EDTAanticoagulated vacutainer tubes and immediately separated, aliquoted, and stored at $-30{ }^{\circ} \mathrm{C}$ in freezers at the township hospital before being couriered $\left(\right.$ at $-30^{\circ} \mathrm{C}$ ) to the Ningxia Medical University central laboratory within $72 \mathrm{~h}$ of blood collection. All plasma samples were subsequently stored at $-80^{\circ} \mathrm{C}$ in the central laboratory. Blood samples will be tested for plasma alpha-linolenic acid (ALA), glucose, LDL-cholesterol, HDL-cholesterol, total cholesterol, and total triglyceride levels. All the specimens will be disposed of 12 months after the end of trial.

Interim visits occurred at 2 weeks and 3 months after the baseline for each participant. Blood test results describing blood lipids were provided to participants at the 2-week study visit, with referral to local physicians for management if blood lipid levels exceeded clinical guidelines. Participants in the intervention groups were reminded to consume walnuts every day and completed a 7-item follow-up questionnaire (Supplementary material) related to acceptability and adherence to the walnut supplementation at both interim visits. Participants in the control group did not answer this questionnaire, but received non-edible gifts at each interim visit. At the end of trial (6 months after randomization), all participants completed the same 24-h dietary recall as they did at baseline to assess the change in nuts intake, and fasting blood samples were collected according to the same procedure as at baseline. At the end of the trial, participants from intervention groups again answered the 7-item questionnaire regarding the acceptability and adherence to walnut supplement, and the control group received a non-edible gift (Table 1 ). All the participants will be notified of the study results once the trial has been completed.

Any condition which required hospitalization was collected at each follow-up visit as adverse event. All adverse events were listed and reported to the ethics committees periodically. Participants who developed 
food allergy with hypersensitivity to any of the components of walnut in the intervention groups were referred to and had the allergy confirmed by a doctor. Intervention was discontinued among those participants but they were followed up until the end of the trial.

Study personnels were divided into an intervention delivery group (who were not blinded to the allocation of treatment) and an outcome assessment group (who were blinded to the allocation of treatment). The intervention delivery group delivered the walnuts and non-edible gifts and performed the interim study visit questionnaires related to acceptability and adherence. The outcome assessors administered the FFQ, anthropometric assessments, and 24-h dietary recall and collected blood samples from participants at baseline and at the end of trial.

\section{Study outcomes}

The primary outcome for the study was fasting plasma alpha-linolenic acid (ALA), which was measured at baseline and 6 months. Walnuts are one of the few dietary sources rich in ALA [30]. Prior studies have demonstrated that increased walnut intake leads to increased plasma ALA, suggesting that measurement of plasma ALA can be used as an objective biomarker to measure adherence to walnut supplementation [10, 31, 32]. Plasma ALA will be assessed using gas chromatography following previously published methods [33-35]. For quality control, identification, precision, and accuracy will be evaluated continuously with model mixtures of known fatty acid methyl esters and an in-house control pool, with identification confirmed by gas chromatography using a flame ionization detector. Based on prior experience, we expect high accuracy and precision for the measurement of ALA (with inter-assay coefficient of variation to be $<10 \%)$. Secondary outcomes included participants' self-reported walnuts intake, adherence and acceptability of the supplement walnuts, as assessed by the 24-h diet recall and 7-item questionnaire. Exploratory outcomes included body weight, plasma lipids (total cholesterol, LDL-C, HDL-C, total triglycerides), and fasting blood glucose.

\section{Sample size}

The sample size of 210 individuals (70 in each arm) provided more than $90 \%$ power with two-sided alpha of 0.05 to detect a mean difference of $0.12 \%$ (as percent of total fatty acid) in plasma ALA between the $30 \mathrm{~g}$ walnut supplementation and control arms [10]. The power estimation assumed a standard deviation (SD) of 0.18 and allowed for a drop-out rate of $10 \%$. The study had similar power to detect a mean difference of $0.12 \%$ in plasma ALA comparing the $60 \mathrm{~g} /$ day and $30 \mathrm{~g} /$ day walnut supplementation groups. The power estimations were conservative since the assumptions were based on the PRED
IMED trial that provided lower doses of walnuts (15 g/ day) than the doses employed in this trial [10].

\section{Statistical analysis}

Differences in between group post-treatment mean plasma ALA will be assessed by linear regression. In the primary analyses, study group assignment will be treated in the ANCOVA model as a categorical independent variable, with the control as the reference group. There will be three pair-wise comparisons: 1) $30 \mathrm{~g} /$ day walnut group vs. control, 2) 60 g/day walnut group vs. control, 3) $60 \mathrm{~g} /$ day walnut group vs. $30 \mathrm{~g} /$ day walnut group. Analyses will adjust for baseline plasma ALA and villages, and follow the intention-to-treat principle. An additional sensitivity analysis will be performed stratified by the baseline CVD status to examine any potential effect modification on the primary outcome.

For the secondary outcome of self-reported mean walnut intake (g/day) assessed by 24-h diet recalls, and the exploratory outcomes (plasma CVD lipids risk factors), differences in end of trial mean values between groups will be assessed by linear regression following the same approach as for the primary outcome.

Self-reported consumption patterns and satisfaction related to walnut intake will be assessed by the 7 -item short questionnaire. The questionnaire included the following items: whether the participants consumed the walnuts, the ways they consumed the walnuts, and the number of times of walnuts intake in a day (each will be summarized as frequencies and \%); as well as the number of days per week the participants consumed walnuts and their satisfaction rating on walnut flavour (will be summarized as mean $\pm \mathrm{SD}$ ).

Statistical significance is defined as two tailed $\alpha=0.05$.

\section{Data management}

The trial data were centrally and securely stored on a Redcap data management server hosted by The George Institute for Global Health, China. Primary collection of screening, intervention, and follow-up visit data were done using the Redcap application on tablets. Quality of the data was ensured by pre-specified range and logic checks built within the Redcap system. All personal information about participants was de-identified and stored in a password protected secured computer.

\section{Participant characteristics}

Participant recruitment started on October 16, 2019 and was completed on October 22, 2019. A total of 246 individuals were screened, and 210 met eligibility criteria and provided informed consent to participate in the trial. Ninety-four percent of participants were recruited on the basis of a history of CVD. Baseline characteristics of the participants were shown in Table 2. The participants 
Table 2 Baseline characteristics of trial participants

\begin{tabular}{|c|c|c|c|c|}
\hline & Total $(n=210)$ & Control $(n=70)$ & Low dose $(n=70)$ & High dose $(n=70)$ \\
\hline Age $(y)$, mean (SD) & $65.4(7.3)$ & $64.4(7.4)$ & $64.7(8.5)$ & $67.0(5.7)$ \\
\hline Female (\%) & 46.7 & 47.1 & 50.0 & 42.9 \\
\hline \multicolumn{5}{|l|}{ Education } \\
\hline Primary school or lower (\%) & 73.3 & 70.0 & 70.0 & 80.0 \\
\hline Junior high school (\%) & 18.1 & 17.1 & 18.6 & 18.6 \\
\hline Senior high school or above (\%) & 8.6 & 12.9 & 11.3 & 1.4 \\
\hline Past smoker (\%) & 44.3 & 44.3 & 40.0 & 48.6 \\
\hline Current smoker(\%) & 15.2 & 17.1 & 11.4 & 17.1 \\
\hline Past drinking (\%) & 34.3 & 35.7 & 34.3 & 32.9 \\
\hline Current drinking (\%) & 4.3 & 2.9 & 2.9 & 7.1 \\
\hline Body mass index $\left(\mathrm{kg} / \mathrm{m}^{2}\right)$, mean (SD) & $26.4(3.4)$ & $26.5(3.4)$ & $25.8(3.6)$ & $26.3(3.1)$ \\
\hline Body weight (kg), mean (SD) & $68.0(11.1)$ & $69.1(11.6)$ & $67.8(12.5)$ & $67.0(9.0)$ \\
\hline Waist hip ratio (SD) & $0.94(0.1)$ & $0.96(0.06)$ & $0.94(0.06)$ & $0.93(0.06)$ \\
\hline Male (SD) & $0.95(0.1)$ & $0.97(0.07)$ & $0.93(0.06)$ & $0.93(0.06)$ \\
\hline Female (SD) & $0.93(0.1)$ & $0.93(0.06)$ & $0.94(0.06)$ & $0.93(0.06)$ \\
\hline Systolic blood pressure (mm Hg), mean (SD) & $147.1(19.9)$ & $146.5(19.9)$ & $146.5(21.8)$ & $146.8(18.1)$ \\
\hline Diastolic blood pressure (mm Hg), mean (SD) & $84.9(12.0)$ & $85.0(12.9)$ & $85.0(12.3)$ & $84.8(10.9)$ \\
\hline \multicolumn{5}{|l|}{ Medication use } \\
\hline Lipid lowering agent (\%) & 67.1 & 55.7 & 78.6 & 67.1 \\
\hline Blood pressure lowering agent (\%) & 85.2 & 84.3 & 80.0 & 91.4 \\
\hline \multicolumn{5}{|l|}{ Disease history } \\
\hline $\operatorname{CVD}^{\mathrm{a}}(\%)$ & 95.2 & 92.9 & 98.6 & 94.3 \\
\hline Stroke (\%) & 31.0 & 24.3 & 25.7 & 42.9 \\
\hline Transient ischemic attack (\%) & 29.5 & 21.4 & 21.4 & 45.7 \\
\hline Ischemic heart disease (\%) & 81.0 & 78.6 & 90.0 & 74.3 \\
\hline Congestive heart failure (\%) & 10.5 & 8.6 & 8.6 & 14.3 \\
\hline Peripheral arterial disease (\%) & 7.6 & 8.6 & 5.7 & 8.6 \\
\hline Hypertension (\%) & 83.3 & 81.4 & 80.0 & 88.6 \\
\hline Diabetes mellitus (\%) & 31.4 & 34.3 & 30.0 & 30.0 \\
\hline
\end{tabular}

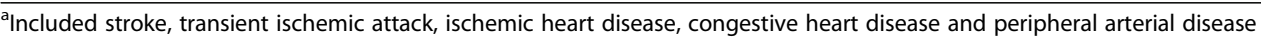

were older adults (mean $[\mathrm{SD}]$ age $=65 \pm 7$ years) and approximately half were female $(47 \%)$. Most participants attained up to primary school education (73\%), with about $60 \%$ of participants were either past or current smokers. Only a small proportion were current drinkers (4.3\%). Most participants had a history of ischemic heart disease (81\%), and many also had a history of stroke (31\%). Participants had similar baseline demographic and medical characteristics across the three randomized groups.

\section{Discussion}

The NUTS study successfully recruited 210 participants in less than 1 week, suggesting that the approach developed in this study can efficiently and rapidly recruit participants at high risk of CVD from rural villages in
China to evaluate a walnut supplementation intervention. The evaluation of the acceptability and adherence to long-term walnut supplementation depended on the complete, unbiased follow up and outcome assessment of participants during the study period. The planned interim follow-ups aimed to engage and motivate participants to adhere to their assigned study group and should help to enhance the integrity of the trial.

The NUTS study was planned to support the design of a future large-scale, long-term, RCT in rural China that will test the effect of increased walnut intake on the risk of CVD. There is a growing recognition that nutrition science will benefit from more adequately-powered and well-conducted RCTs to evaluate the health effects of nutrients and foods on clinical outcomes, which can complement findings from prospective cohorts and 
metabolic studies [36]. These research approaches have different strengths and weaknesses, and consistency of findings can significantly enhance the development of dietary guidelines and their effective implementation. For instance, the seafood-derived, long-chain omega-3 polyunsaturated fatty acids have been shown to associate with lower risk of coronary heart disease (CHD) death in observational cohort studies, and reduce CHD deaths in several adequately powered RCTs $[37,38]$. This coherent evidence base has important implications for current dietary and clinical guidelines to include omega-3 rich seafood for the prevention of coronary heart disease and omega-3 fatty acid supplement for the secondary prevention of CHD [9, 37, 39]. Our future planned RCT aims to similarly provide a critical part of the evidence base for nuts and cardiovascular health.

There were several notable strengths of the NUTS pilot study. First, this 3-arm randomized controlled trial allowed testing for a potential dose-response effect of walnut consumption by including two intervention groups. Second, the trial had sufficient power, and conservatively assumed an effect size based on that observed in the PREDIMED trial, which used a lower dose $(15 \mathrm{~g} /$ day) of walnuts together with a mixture of $7.5 \mathrm{~g} /$ day of almonds and $7.5 \mathrm{~g} /$ day of hazelnuts [10]. Third, using plasma ALA as an objective biomarker to assess the primary outcome of adherence minimized the risk of measurement bias and errors associated with self-reported dietary intake. We also maintained blinding of all outcome assessors to further safeguard against ascertainment bias. Finally, the 6-month intervention duration was longer than the majority of previous trials, and provided evidence on the feasibility of nuts supplementation over an extended period $[1,2]$.

There were also limitations to this study. Firstly, participants were adults at high risk of CVD from rural Chinese villages, and findings of acceptability and adherence to walnut supplementation may not be generalizable to other populations. However, because the main purpose of the study was to aid the design and conduct of an RCT done in the same population, this was not a major concern. Secondly, this trial used a pragmatic approach with broad inclusion criteria that cover both individuals at high risk of CVD (without prior CVD) and those with previous CVD. These criteria were chosen to provide evidence about the feasibility of recruitment of participants for a future large trial (anticipated $>20,000$ ) which would target both primary and secondary prevention. Such broad inclusion criteria are common for cardiovascular outcome trials [40, 41] where effects of interventions are typically homogenous across the groups. Increased nuts intake, for example, reduces LDL-cholesterol, which could be expected to contribute to both primary and secondary prevention
$[42,43]$. Finally, given the nature of the intervention, it was not possible to blind the participants to their randomized group, and there was a risk of bias as a consequence. To address this, outcome assessors were blinded to the group allocation and the primary outcome was an objective blood assay. It was possible that those in the control group may have altered their walnut intake if they became aware of the trial focus, and we evaluated this possibility using the dietary questionnaire surveys. However, sustained, daily increased intake of walnuts without provision appeared unlikely due to the relatively high price of walnuts compared to other commonly consumed foods in the area.

\section{Conclusions}

In conclusion, the NUTS trial successfully recruited the targeted number of participants at high risk of CVD. The result of this trial will provide novel evidence to support the acceptability and adherence to long-term walnut supplementation in this population and will inform the design of a future CVD event-driven trial to test the effect of walnut supplementation for CVD prevention.

\section{Supplementary Information}

The online version contains supplementary material available at https://doi. org/10.1186/s12937-020-00660-7.

\section{Additional file 1}

\section{Abbreviations}

CVD: Cardiovascular disease; ALA: Alpha linolenic acid; RCT: Randomized controlled trial; FFQ: Food frequency questionnaire

\section{Acknowledgements}

We gratefully acknowledge the contribution of all the village doctors and participants in the NUTS study for their diligence and cooperation.

\section{Disclosures}

M. Huffman has received grant support from the World Heart Federation via Boehringer Ingelheim and Novartis; the American Heart Association, Verily, and AstraZeneca; and the American Medical Association for work unrelated to this paper. He notes institutional relationships through his appointment at The George Institute with AbbVie, Actelion, and Janssen.

\section{Authors' contributions}

JW and $Y Z$ directed the study and had primary responsibility for the content; $Y L$ and NL conducted the study, analysed the data, and $Y L$ drafted the paper; QL advised the analysis and interpretation of data; BN and MT supervised the study and substantially revised the work; NL, NY, XP, RM, DM, $\mathrm{MH}, \mathrm{YW}, \mathrm{YZ}$ critically revised the article. All authors have read and approved the final manuscript.

\section{Funding}

The study was supported by a National Health and Medical Research Council of Australia (NHMRC) Program Grant (APP1149987). The authors are solely responsible for the design and conduct of this study, all study analyses, and the drafting and editing of the manuscript and its contents.

Availability of data and materials

The datasets used and analysed during the current study are available from the corresponding author on reasonable request but restrictions 
apply to the availability of participants' individual data under the Chinese data protection law.

\section{Ethics approval and consent to participate}

The trial has been registered in the ClinicalTrials.gov database on July 30 2019 (registration number: NCT04037943, https://clinicaltrials.gov/ct2/show/ NCT04037943?term=NCT04037943\&draw=2\&rank=1). The study was approved by the institutional review board of Ningxia Medical University and the Human Research Ethics Committee at the University of New South Wales. A data monitoring committee (DMC) was not convened in this shortterm trial since the main objective was to assess feasibility and acceptability of the intervention.

\section{Consent for publication}

Not applicable.

\section{Competing interests}

The authors declare that they have no competing interests.

\section{Author details}

${ }^{1}$ The George Institute for Global Health, Faculty of Medicine, University of New South Wales, Sydney, Australia. ${ }^{2}$ The George Institute for Global Health at Peking University Health Science Center, Beijing, China. ${ }^{3}$ Ningxia Medical University, Yinchuan, China. ${ }^{4}$ Friedman School of Nutrition Science and Policy, Tufts University, Boston, MA, USA. ${ }^{5}$ Department of Preventive Medicine, Feinberg School of Medicine, Northwestern University, Chicago, IL, USA. ${ }^{6}$ Peking University Clinical Research Institute, Beijing, China. ${ }^{7}$ School of Public Health, Imperial College London, London, UK. ${ }^{8}$ Sydney School of Public Health, University of Sydney, Sydney, Australia.

Received: 11 February 2020 Accepted: 15 December 2020 Published online: 06 January 2021

\section{References}

1. Del Gobbo LC, Falk MC, Feldman R, Lewis K, Mozaffarian D. Effects of tree nuts on blood lipids, apolipoproteins, and blood pressure: systematic review, meta-analysis, and dose-response of 61 controlled intervention trials. Am J Clin Nutr. 2015;102(6):1347-56.

2. Neale EP, Tapsell LC, Guan V, Batterham MJ. The effect of nut consumption on markers of inflammation and endothelial function: a systematic review and meta-analysis of randomised controlled trials. BMJ Open. 2017;7(11): e016863.

3. Viguiliouk E, Kendall CW, Blanco Mejia S, Cozma Al, Ha V, Mirrahimi A, et al. Effect of tree nuts on glycemic control in diabetes: a systematic review and meta-analysis of randomized controlled dietary trials. PLoS One. 2014;9(7): e103376.

4. Imamura F, Micha R, Wu JH, de Oliveira Otto MC, Otite FO, Abioye Al, et al. Effects of saturated fat, polyunsaturated fat, monounsaturated fat, and carbohydrate on glucose-insulin homeostasis: a systematic review and meta-analysis of randomised controlled feeding trials. J PLoS Med. 2016; 13(7):e1002087.

5. Ros E, Mataix J. Fatty acid composition of nuts - implications for cardiovascular health. Br J Nutr. 2006;96(S2):S29-35.

6. Aune D, Keum N, Giovannucci E, Fadnes LT, Boffetta P, Greenwood DC, et al. Nut consumption and risk of cardiovascular disease, total cancer, allcause and cause-specific mortality: a systematic review and dose-response meta-analysis of prospective studies. BMC Med. 2016;14(1):207.

7. Wang S-s, Lay S, Yu H-n, Shen S-r. Dietary guidelines for Chinese residents (2016): comments and comparisons. J Zhejiang Univ Sci B. 2016;17(9):649-56.

8. Binns CW, Lee MK, Kagawa M, Low WY, Liqian Q, Guldan GS, et al. Dietary guidelines for the Asia Pacific region: report of the Asia Pacific academic consortium for public health workshop 2016. Asia Pacific J Public Health. 2017;29(2):98-101.

9. Department of Health and Human Services (US), Department of Agriculture (US). 2015-2020 dietary guidelines for Americans. 8th ed; 2015. [cited 2020 July 7 ]. Available from: http://health.gov/dietaryguidelines/2015/guidelines.

10. Estruch R, Ros E, Salas-Salvadó J, Covas M-I, Corella D, Arós F, et al. Primary Prevention of Cardiovascular Disease with a Mediterranean Diet Supplemented with Extra-Virgin Olive Oil or Nuts. N Engl J Med. 2018; 378(25):e34
11. Agarwal A, loannidis JPA. PREDIMED trial of Mediterranean diet: retracted, republished, still trusted? BMJ. 2019;364:1341.

12. Li SC, Liu YH, Liu JF, Chang WH, Chen CM, Chen CY. Almond consumption improved glycemic control and lipid profiles in patients with type 2 diabetes mellitus. Metabolism. 2011;60(4):474-9.

13. Wang $X, L i$ Z, Liu Y, Lv X, Yang W. Effects of pistachios on body weight in Chinese subjects with metabolic syndrome. Nutr J. 2012;11:20.

14. Wu H, Pan A, Yu Z, Qi Q, Lu L, Zhang G, et al. Lifestyle counseling and supplementation with flaxseed or walnuts influence the management of metabolic syndrome. J Nutr. 2010;140(11):1937-42.

15. Mohan V, Gayathri R, Jaacks LM, Lakshmipriya N, Anjana RM, Spiegelman D, et al. Cashew nut consumption increases HDL cholesterol and reduces systolic blood pressure in Asian Indians with type 2 diabetes: a 12-week randomized controlled trial. J Nutr. 2018;148(1):63-9.

16. Lee YJ, Nam GE, Seo JA, Yoon T, Seo I, Lee JH, et al. Nut consumption has favorable effects on lipid profiles of Korean women with metabolic syndrome. Nutr Res. 2014;34(9):814-20.

17. Liu S, Li Y, Zeng $X$, Wang H, Yin P, Wang L, et al. Burden of cardiovascular diseases in China, 1990-2016: findings from the 2016 global burden of disease study. JAMA Cardiol. 2019;4(4):342-52.

18. Li Y, Wang DD, Ley SH, Vasanti M, Howard AG, He Y, et al. Time trends of dietary and lifestyle factors and their potential impact on diabetes burden in China. Diabetes Care. 2017:40(12):1685-94.

19. Zhang G, Yu C, Zhou M, Wang L, Zhang Y, Luo L. Burden of Ischaemic heart disease and attributable risk factors in China from 1990 to 2015: findings from the global burden of disease 2015 study. BMC Cardiovasc Disord. 2018;18(1):18.

20. Zhou M, Wang H, Zhu J, Chen W, Wang L, Liu S, et al. Cause-specific mortality for 240 causes in China during 1990-2013: a systematic subnational analysis for the global burden of disease study 2013. Lancet. 2016;387(10015):251-72.

21. Zhao D, Liu J, Wang M, Zhang X, Zhou M. Epidemiology of cardiovascular disease in China: current features and implications. Nat Rev Cardiol. 2019; 16(4):203-12.

22. Neal B, Perkovic V, de Zeeuw D, Mahaffey KW, Fulcher G, Stein P, et al. Rationale, design, and baseline characteristics of the Canagliflozin Cardiovascular Assessment Study (CANVAS)--a randomized placebocontrolled trial. Am Heart J. 2013;166(2):217-23.e11.

23. Neal B, Tian M, Li N, Elliott P, Yan LL, Labarthe DR, et al. Rationale, design, and baseline characteristics of the salt substitute and stroke study (SSaSS) - a large-scale cluster randomized controlled trial. Am Heart J. 2017; 188:109-17.

24. Ros E. Health benefits of nut consumption. Nutrients. 2010;2(7):652-82.

25. Hayes D, Angove MJ, Tucci J, Dennis C. Walnuts (Juglans regia) chemical composition and research in human health. Crit Rev Food Sci Nutr. 2016; 56(8):1231-41.

26. Guasch-Ferré M, Li J, Hu FB, Salas-Salvadó J, Tobias DK. Effects of walnut consumption on blood lipids and other cardiovascular risk factors: an updated meta-analysis and systematic review of controlled trials. Am J Clin Nutr. 2018;108(1):174-87

27. Tindall AM, Johnston EA, Kris-Etherton PM, Petersen KS. The effect of nuts on markers of glycemic control: a systematic review and meta-analysis of randomized controlled trials. Am J Clin Nutr. 2019;109(2):297-314.

28. Ramos DE. Walnut production manual: UCANR publications; 1997.

29. Li Y, He Y, Zhai F, Yang X, Hu X, Zhao W, et al. Comparison of assessment of food intakes by using 3 dietary survey methods. Chin J Prev Med. 2006;04:273-80.

30. Mozaffarian D, Wu JH. Omega-3 fatty acids and cardiovascular disease: effects on risk factors, molecular pathways, and clinical events. J Am Coll Cardiol. 2011:58(20):2047-67.

31. Iwamoto M, Imaizumi K, Sato M, Hirooka Y, Sakai K, Takeshita A, et al. Serum lipid profiles in Japanese women and men during consumption of walnuts. Eur J Clin Nutr. 2002;56:629.

32. Rock CL, Flatt SW, Barkai H-S, Pakiz B, Heath DD. Walnut consumption in a weight reduction intervention: effects on body weight, biological measures, blood pressure and satiety. Nutr J. 2017;16(1):76.

33. Ohta A, Mayo MC, Kramer N, Lands WEM. Rapid analysis of fatty acids in plasma lipids. Lipids. 1990;25(11):742-7.

34. Cruz-Hernandez C, Thakkar SK, Masserey-Elmelegy I, Buosi W, Fontannaz P, Giuffrida F. Quantification of fatty acids in erythrocytes and plasma by fast gas chromatography. J Sep Sci. 2017:40(16):3289-300. 
35. Bondia-Pons I, Castellote Al, López-Sabater MC. Comparison of conventional and fast gas chromatography in human plasma fatty acid determination. J Chromatogr B. 2004;809(2):339-44.

36. Trepanowski JF, loannidis JPA. Perspective: limiting dependence on nonrandomized studies and improving randomized trials in human nutrition research: why and how. Adv Nutr. 2018;9(4):367-77.

37. Siscovick DS, Barringer TA, Fretts AM, Wu JH, Lichtenstein AH, Costello RB, et al. Omega-3 polyunsaturated fatty acid (fish oil) supplementation and the prevention of clinical cardiovascular disease: a science advisory from the American Heart Association. Circulation. 2017;135(15):e867-e84.

38. Wu JHY, Micha R, Mozaffarian D. Dietary fats and cardiometabolic disease: mechanisms and effects on risk factors and outcomes. Nat Rev Cardiol. 2019;16(10):581-601.

39. Rimm Eric B, Appel Lawrence J, Chiuve Stephanie E, Djoussé L, Engler Mary B, Kris-Etherton Penny M, et al. Seafood long-chain n-3 polyunsaturated fatty acids and cardiovascular disease: a science advisory from the American Heart Association. Circulation. 2018;138(1):e35-47.

40. Yokoyama M, Origasa H, Matsuzaki M, Matsuzawa Y, Saito Y, Ishikawa Y, et al. Effects of eicosapentaenoic acid on major coronary events in hypercholesterolaemic patients (JELIS): a randomised open-label, blinded endpoint analysis. Lancet. 2007;369(9567):1090-8.

41. Howard BV, Van Horn L, Hsia J, Manson JE, Stefanick ML, Wassertheil-Smoller $\mathrm{S}$, et al. Low-fat dietary pattern and risk of cardiovascular disease: the Women's Health Initiative Randomized Controlled Dietary Modification Trial. JAMA. 2006;295(6):655-66.

42. Brugts JJ, Yetgin T, Hoeks SE, Gotto AM, Shepherd J, Westendorp RGJ, et al. The benefits of statins in people without established cardiovascular disease but with cardiovascular risk factors: meta-analysis of randomised controlled trials. BMJ. 2009;338:b2376

43. Baigent C, Keech A, Kearney PM, Blackwell L, Buck G, Pollicino C, et al. Efficacy and safety of cholesterol-lowering treatment: prospective metaanalysis of data from 90,056 participants in 14 randomised trials of statins. Lancet (London, England). 2005;366(9493):1267.

\section{Publisher's Note}

Springer Nature remains neutral with regard to jurisdictional claims in published maps and institutional affiliations.

Ready to submit your research? Choose BMC and benefit from:

- fast, convenient online submission

- thorough peer review by experienced researchers in your field

- rapid publication on acceptance

- support for research data, including large and complex data types

- gold Open Access which fosters wider collaboration and increased citations

- maximum visibility for your research: over $100 \mathrm{M}$ website views per year

At $\mathrm{BMC}$, research is always in progress.

Learn more biomedcentral.com/submissions 